${ }^{6}$ Duke-NUS Medical School, SingHealth Duke-NUS Medicine Academic Clinical Programme, Singapore, Singapore; ${ }^{7}$ Genome Institute of Singapore (GIS), Genome Institute of Singapore, Singapore, Singapore; ${ }^{8}$ Singapore General Hospital, Pathology, Singapore, Singapore; ${ }^{9}$ Duke-NUS Medical School, Cancer \& Stem Cell Biology, Singapore, Singapore; ${ }^{10}$ National Cancer Centre Singapore, National Cancer Centre Singapore, Singapore, Singapore

Background: Systemic lupus erythematosus (SLE) is a multi-organ autoimmune disease that is potentially fatal. There is an unmet need to improve current therapies. In patients with SLE, we observed that serum CXCL5 levels were significantly lower than healthy control subjects and negatively correlated with disease activity ${ }^{(1-9)}$

Objectives: The aim of this study is to elucidate the effect of supplemental serum CXCL5 in abrogating the pathological processes of SLE.

Methods: Ten doses of exogenous CXCL5 $(3 \mu \mathrm{g} / \mathrm{kg})$ was administered to 16-week-old Fas ${ }^{\text {Ipr }}$ mice weekly by intravenous injection. Mice were monitored for 10 weeks. Splenic immune profile was measured by flow cytometry. Circulating cytokine and immunoglobulin profile were detected by Luminex technology. Renal function was evaluated by urinary spot albumin creatinine ratio. In situ renal immune cell infiltration and complement 3 deposition were detected by Haematoxylin and Eosin (H\&E) and immunohistochemistry staining. The molecular pathways involved were examined by RNA sequencing.

Results: In Fas ${ }^{\text {Ipr }}$ mice, intravenous administration of exogenous CXCL5 significantly improved mouse survival with concomitant reduction of autoantibody secretion, proteinuria, complement 3 deposition, neutrophil infiltration and lupus nephritis classes. Through evaluating the changes of immune profile, cytokine profile and molecular pathways, we found that intravenous CXCL5 reduced inflammation via an orchestral effect of regulating neutrophil trafficking and modulating helper T cell-mediated immune response. Pharmacokinetic and real-time Polymerase Chain Reaction studies further demonstrated that this orchestration was triggered by a cascade reaction - restoring vascular under-expressed CXCL5 by an exogenous stimulation, re-establishing the normal serum levels of endogenous CXCL5 and reverting the CXCL5 chemokine gradient between inflamed tissues and blood circulation.

Conclusion: Managing the dysregulation of CXCL5 by exogenous supplement may provide a new option for SLE therapy.

REFERENCES:

[1] Dufies M, Grytsai O, Ronco C, et al. New CXCR1/CXCR2 inhibitors represent an effective treatment for kidney or head and neck cancers sensitive or refractory to reference treatments. Theranostics. 2019;9(18):5332-5346. doi:10.7150/thno.34681

[2] Yildirim K, Colak E, Aktimur R, et al. Clinical Value of CXCL5 for Determining of Colorectal Cancer. Asian Pac J Cancer Prev. Sep 26 2018;19(9):24812484. doi:10.22034/apjcp.2018.19.9.2481

[3] Wu K, Yu S, Liu Q, Bai X, Zheng X. The clinical significance of CXCL5 in nonsmall cell lung cancer. Onco Targets Ther. 2017;10:5561-5573. doi:10.2147/ ott.s148772

[4] Zhao J, Ou B, Han D, et al. Tumor-derived CXCL5 promotes human colorectal cancer metastasis through activation of the ERK/Elk-1/Snail and AKT/ GSK3beta/beta-catenin pathways. Mol Cancer. Mar 29 2017;16(1):70. doi:10.1186/s12943-017-0629-4

[5] Han KQ, Han H, He XQ, et al. Chemokine CXCL1 may serve as a potential molecular target for hepatocellular carcinoma. Cancer Med. Oct 2016;5(10):2861-2871. doi:10.1002/cam4.843

[6] Pappa CA, Tsirakis G, Kanellou P, et al. Monitoring serum levels ELR+ CXC chemokines and the relationship between microvessel density and angiogenic growth factors in multiple myeloma. Cytokine. Dec 2011;56(3):616-20. doi:10.1016/j.cyto.2011.08.034

[7] Zhang L, Li H, Ge C, et al. CXCL3 contributes to CD133(+) CSCs maintenance and forms a positive feedback regulation loop with CD133 in HCC via Erk1/2 phosphorylation. Sci Rep. Jun 3 2016;6:27426. doi:10.1038/ srep27426

[8] Matsubara J, Honda K, Ono M, et al. Reduced plasma level of CXC chemokine ligand 7 in patients with pancreatic cancer. Cancer Epidemiol Biomarkers Prev. Jan 2011;20(1):160-71. doi:10.1158/1055-9965.epi-10-0397

[9] Ma Y, Ren Y, Dai ZJ, Wu CJ, Ji YH, Xu J. IL-6, IL-8 and TNF-alpha levels correlate with disease stage in breast cancer patients. Adv Clin Exp Med. May-Jun 2017;26(3):421-426. doi:10.17219/acem/62120

Disclosure of Interests: None declared

DOI: 10.1136/annrheumdis-2021-eular.2262

\section{POS0418 \\ SPLENIC EXTRAMEDULLARY HEMATOPOIESIS IS OMNIPRESENT AND CORRELATES WITH DISEASE SEVERITY IN THE LUPUS NZB/W F1 MURINE MODEL}

S. Doumas ${ }^{1,2}$, M. Grigoriou ${ }^{1}$, A. Banos ${ }^{1,3}$, C. Giakopoulou 4 , I. Mitroulis ${ }^{5}$, P. Verginis ${ }^{6}$, D. Boumpas ${ }^{1,2,7} .{ }^{1}$ Biomedical Research Foundation of the Academy of Athens, Laboratory of Inflammation and Autoimmunity, Athens, Greece; ${ }^{2}$ Attikon University Hospital and Joint Rheumatology Program, National and
Kapodistrian University of Athens, 4th Department of Internal Medicine, Athens, Greece; ${ }^{3}$ Hippokration General Hospital, 2nd Department of Internal Medicine, Athens, Greece; ${ }^{4}$ Medical School, National and Kapodistrian University of Athens, 1 st Department of Pathology, Athens, Greece; ${ }^{5}$ Democritus University of Thrace, Department of Hematology and Laboratory of Molecular Hematology, Department of Medicine, Alexandroupoli, Greece; ${ }^{6}$ University of Crete Medical School, Laboratory of Immune Regulation and Tolerance, Heraklion, Greece; ${ }^{7}$ Medical School, University of Cyprus, Rheumatology-Clinical Immunology Unit, Nicosia, Greece

Background: Extramedullary hematopoiesis (EMH) is increasingly recognized as an integral component of systemic inflammatory diseases; compared to their bone marrow counterparts, hematopoietic progenitors of EMH have an enhanced role in target organ damage ${ }^{1,2}$. We have found that $\beta$-glucan -a non-specific inducer of reprograming of innate immunity- results in dramatic EMH with marked increase in Long-Term (LT)-HSCs, massive splenomegaly and worsening of nephritis in the NZB/W F1 lupus murine model (unpublished data) Objectives: To investigate EMH's time course and contribution to inflammatory target-organ damage (kidney) in the NZB/W F1 lupus murine model.

Methods: Spleens and kidneys were isolated from female NZB/W F1, at pre-nephritic stage (3-month-old) and nephritic stage (>6-month-old), and age/sex matched C57BL/6 (WT controls). Single-cell suspensions of spleens were analyzed by flow cytometry for Hematopoietic Stem and progenitor cells (HSPCs) phenotyping. Formalin-fixed and paraffin-embedded sections of spleens and kidneys were stained with conventional histological stains (H\&E, Silver, Trichrome Masson). Spleens were histologically assessed for the presence of EMH and kidneys were assessed for activity and chronicity through the NIH Lupus nephritis scoring system.

Results: Histological analysis revealed that NZW/B F1 mice at the nephritic stage display massive splenomegaly with concomitant expansion of the red pulp, increased presence of megakaryocytes and disorganized splenic architecture. This is further corroborated by the flow cytometry analysis which demonstrated a significant increase of all HSPCs subsets (Long-term/Short-term Hematopoietic Stem Cells and Multipotent progenitors) compared to the C57BL/6 WT controls at nephritic stage. The degree of HSPC expansion and splenic architecture disorganization correlates strongly with the activity of lupus nephritis as quantified by the NIH scoring system. Of note, evidence of splenic EMH were present even in 3-month-old animals before overt nephritis ensues.

Conclusion: Extramedullary hematopoiesis is present before overt nephritis and is dramatically expanded at the nephritic stage of the NZW/B F1 mouse model. The degree of EMH positively correlates with the severity of lupus nephritis. These data support a pathogenic role of EMH, and spleen derived HSPCs, in driving lupus nephritis.

\section{REFERENCES:}

[1] Regan-Komito, D., Swann, J.W., Demetriou, P., Cohen, E.S., Horwood, N.J., Sansom, S.N., Griseri, T., 2020. GM-CSF drives dysregulated hematopoietic stem cell activity and pathogenic extramedullary myelopoiesis in experimental spondyloarthritis. Nature Communications 11, 155

[2] Griseri, T., McKenzie, B.S., Schiering, C., Powrie, F., 2012. Dysregulated Hematopoietic Stem and Progenitor Cell Activity Promotes Interleukin-23-Driven Chronic Intestinal Inflammation. Immunity 37, 1116-1129.

Acknowledgements: This project has received funding from the European Research Council (ERC) under the European Union's Horizon 2020 research and innovation programme (grant agreement No 742390)

Disclosure of Interests: None declared

DOI: 10.1136/annrheumdis-2021-eular.2490

\section{\begin{tabular}{|l|l}
\hline POS0419 ABERRANT SPLICEOSOME AND ALTERED \\
\hline
\end{tabular} EXPRESSION OF IFN-RESPONSE RELATED GENES ARE HALLMARKS OF MONOCYTES FROM LUPUS PATIENTS WITH RENAL DISEASE}

A. M. Patiño-Trives ${ }^{1}$, C. Perez-Sanchez ${ }^{1}$, A. Ibañez-Costa ${ }^{2}$, M. Luque-Tévar ${ }^{1}$ M. D. C. Abalos-Aguilera ${ }^{1}$, I. Arias de la Rosa ${ }^{1}$, D. Ruiz' ${ }^{1}$, P. Seguí Azpilcueta ${ }^{1}$ M. Espinosa ${ }^{1}$, N. Barbarroja Puerto ${ }^{1}$, J. P. Castaño ${ }^{2}$, R. M. Luque Huertas ${ }^{2}$, M. A. Aguirre ${ }^{1}$, C. Lopez-Pedrera ${ }^{3} .{ }^{1}$ IMIBIC, Reina Sofia Hospital/University of Cordoba, Cordoba, Spain, Córdoba, Spain: ${ }^{2} I M I B I C$, Department of Cell Biology, Physiology and Immunology, University of Cordoba, Spain, Córdoba, Spain: ${ }^{3}$ IMIBIC, Reina Sofia Hospital/University of Cordoba, Cordoba, Spain, Cordoba, Spain

Background: To date, novel mechanisms such as the involvement of splicing machinery components in lupus nephropathy and its interplay with the transcriptome in innate immune cells have not been evaluated.

Objectives: 1- To identify altered transcriptomic signatures associated with the immune response of monocytes from SLE patients and its association with clinical features. 2- To evaluate the role of the spliceosome linked to the transcriptomic profile of SLE monocytes. 3- To analyze mechanistically the impact of 